Comunicação Breve

Brief Communication

Aparecido José Couto Soares ${ }^{1}$

Seisse Gabriela Gandolfi Sanches ${ }^{1}$

Débora Cristina Alves ${ }^{1}$

Renata Mota Mamede Carvallo ${ }^{1}$

Maria Silvia Cárnio ${ }^{1}$

Descritores

Avaliação

Aprendizagem

Leitura

Audição

Transtornos de aprendizagem

Keywords

Evaluation

Learning

Reading

Hearing

Learning disorders

Endereço para correspondência:

Aparecido José Couto Soares

R. Cipotânea, 51, Cidade Universitária,

São Paulo (SP), Brasil, CEP: 05360-160.

E-mail: ajcsoares@usp.br

Recebido em: 16/8/2012

Aceito em: 8/1/2013

\section{Processamento temporal e consciência fonológica nas alterações de leitura e escrita: dados preliminares}

\author{
Temporal auditory processing and phonological awareness \\ in reading and writing disorders: preliminary data
}

\section{RESUMO}

Objetivo: Verificar se há associação entre o desempenho nos testes auditivos temporais e na consciência fonológica em indivíduos com alterações de leitura e escrita. Métodos: Foram sujeitos deste estudo 16 crianças, entre 7 e 12 anos, com alterações de leitura e escrita confirmada após avaliação específica. Todos os participantes foram submetidos à avaliação da consciência fonológica, utilizando-se o teste de consciência fonológica - Instrumento de Avaliação Sequencial. Para avaliar o processamento auditivo temporal foram utilizados os testes de padrão de duração e frequência, realizados em cabina audiométrica. Resultados: A análise descritiva indicou desempenho alterado nas habilidades silábicas e fonêmicas da consciência fonológica, bem como nos testes temporais. O teste de Fisher indicou associação entre a presença de alteração no processamento temporal e nas tarefas de consciência fonológica $(\mathrm{p}<0,001)$, sugerindo que a alteração no processamento temporal contribua para pior desempenho nestas habilidades. Conclusões: Houve associação entre o desempenho em testes auditivos temporais e consciência fonológica. Os dados encontrados trazem reflexões no sentido de incluir a avaliação dos padrões temporais dentre os procedimentos utilizados na avaliação dos indivíduos com alterações de leitura e escrita.

\begin{abstract}
Purpose: To verify if there is an association between temporal auditory tests and phonological awareness in individuals with reading and writing disorders. Methods: Sixteen children were subjects of this study, aged between 7 and 12 years old, who had reading and writing disorders confirmed after specific assessment. All participants underwent phonological awareness assessment using CONFIAS test. In order to assess the auditory temporal processing, duration and frequency pattern tests were used. Results: The descriptive analysis indicated low performance in syllabic and phonemic activities of phonological awareness as well as in temporal auditory tests. Fisher's test indicated association between disorders in auditory temporal processing and phonological awareness $(\mathrm{p}>0.001)$. It suggests that disorders in temporal processing contribute to low performance in phonological awareness tasks. Conclusions: There was association between performance in temporal auditory tests and in the phonological awareness. Data found provide reflections about including temporal auditory assessment among procedures used in the analysis of individuals with reading and writing disorders.
\end{abstract}

Trabalho realizado nos Laboratórios de Investigação Fonoaudiológica em Leitura e Escrita e de Investigação Fonoaudiológica em Audição Humana do Departamento de Fisioterapia, Fonoaudiologia e Terapia Ocupacional, Faculdade de Medicina, Universidade de São Paulo - USP - São Paulo (SP), Brasil.

(1) Departamento de Fisioterapia, Fonoaudiologia e Terapia Ocupacional, Faculdade de Medicina, Universidade de São Paulo - FMUSP - São Paulo (SP), Brasil.

Conflito de interesse: nada a declarar. 


\section{INTRODUÇÃO}

Alterações no processamento temporal podem levar a um baixo desempenho nas habilidades de leitura e escrita ${ }^{(1,2)}$. A proficiência na manipulação dos sons da fala tem relação com o melhor desempenho em leitura. Tal habilidade caracteriza a consciência fonológica $(\mathrm{CF})^{(3-5)}$, geralmente prejudicada nas alterações de leitura e escrita. Estudos ${ }^{(6-9)}$ afirmam que indivíduos com alterações de leitura e escrita podem apresentar atraso no desenvolvimento das habilidades auditivas, impedindo $o$ adequado processamento da informação e interferindo no processo de aprendizagem.

Considerando que o processamento temporal está relacionado à percepção da fala ${ }^{(10)}$, é importante a aplicação de testes auditivos envolvendo habilidades temporais nas alterações das linguagens oral e escrita. Salienta-se a necessidade de investigar a relação entre o desempenho em $\mathrm{CF}$ e testes auditivos temporais, pois a integridade dos mecanismos fisiológicos auditivos tem papel fundamental na aquisição da leitura e da escrita ${ }^{(11)}$. Assim, o objetivo deste estudo foi verificar se há associação entre o desempenho em testes auditivos temporais e em $\mathrm{CF}$ em indivíduos com alterações de leitura e escrita.

\section{MÉTODOS}

Estudo iniciado após a aprovação do Comitê de Ética da Faculdade de Medicina da Universidade de São Paulo (USP), $\operatorname{sob~}^{\circ}$ 305/10. Foram estudados 16 pacientes ( 7 a 12 anos, com média de idade de 10 anos e 6 meses) com alterações de leitura e da escrita, sendo 5 do gênero feminino e 11 do masculino. Os critérios de inclusão foram: estar em atendimento fonoaudiológico; não apresentar sinais e sintomas de distúrbios neurológicos, comportamentais ou cognitivos e limiares auditivos tonais $\leq 20$ dBNA. Todas as crianças foram submetidas à avaliação audiológica (audiometria tonal e vocal). Os pacientes passaram por avaliação específica de leitura, escrita e habilidades subjacentes a elas, tais como nomeação automática rápida, $\mathrm{CF}$ e memória operacional fonológica, aplicadas pelos pesquisadores.

Para a avaliação da CF, aplicou-se a prova de CF Instrumento de Avaliação Sequencial (CONFIAS) ${ }^{(12)}$, que foi realizada individualmente, conforme instruções descritas no teste $^{(12)}$. A pontuação máxima é 70 (40 pontos - silábica e 30 fonêmica). O desempenho dos sujeitos foi classificado como esperado/alterado, considerando a classificação descrita no teste que estabelece o número de acertos esperado para cada criança, conforme seu nível de escrita ${ }^{(12)}$.

A fim de avaliar o processamento auditivo temporal, foram utilizados os testes de padrão de duração (TPD) e frequência (TPF), AUDITEC (Saint Louis, USA), realizados em cabina audiométrica, aplicados em ambas as orelhas ao mesmo tempo na intensidade de $40 \mathrm{dBNS}$ sobre o valor do limiar de reconhecimento de fala, com o auxílio de um CD-player conectado ao audiômetro GSI 61 (GSI Grason Stadler, Eden Prairie, MN, USA).

Os dados foram submetidos à análise estatística. Para verificar a associação entre as variáveis do estudo foi utilizado o teste exato de Fisher, com nível de significância de 0,05.

\section{RESULTADOS}

A análise descritiva indicou desempenho alterado nas habilidades silábicas e fonêmicas (Tabela 1).

Tabela 1. Desempenho dos sujeitos nos testes de consciência fonológica

\begin{tabular}{lccccc}
\hline $\begin{array}{l}\text { Tarefas } \\
\text { de consciência } \\
\text { fonológica }\end{array}$ & Média & $\begin{array}{l}\text { Desvio } \\
\text { padrão }\end{array}$ & Mediana & Mínimo & Máximo \\
\hline Sílabas & 34,75 & 7,73 & 37,00 & 9,00 & 40,00 \\
Fonemas & 23,25 & 7,67 & 27,00 & 8,00 & 30,00 \\
\hline
\end{tabular}

O teste de Fisher apontou associação entre a presença de alteração no processamento temporal e nas tarefas de CF ( $\mathrm{p}<0,001$ ), sugerindo que a alteração do processamento auditivo temporal contribuiu para o pior desempenho nas habilidades de CF (Tabela 2).

Tabela 2. Desempenho dos sujeitos de acordo com a presença de alteração nos testes auditivos temporais e o desempenho na consciência fonológica $(n=16)$

\begin{tabular}{lccccc}
\hline & & \multicolumn{4}{c}{ Consciência fonológica } \\
\cline { 2 - 6 } & & Esperado & Alterado & Total & Valor p \\
\hline Presença de & Não & 5 & 0 & 5 & \\
$\begin{array}{l}\text { alteração } \\
\text { de ordenação }\end{array}$ & Sim & 0 & 11 & 11 & $<0,001^{*}$ \\
temporal & Total & 5 & 11 & 16 & \\
\hline
\end{tabular}

\section{DISCUSSÃO}

O processamento temporal consiste na percepção de características temporais que auxiliam na identificação de pequenas variações acústicas, as quais ocorrem no sinal de fala e permitem que o indivíduo realize distinções segmentais, silábicas e de palavras na fala contínua ${ }^{(13)}$. Dessa forma, pode-se afirmar que o desenvolvimento das habilidades do processamento temporal é importante para a CF, uma vez que esta envolve tarefas de síntese, segmentação e transposição silábica e fonêmica.

Ao considerar que o processamento temporal está relacionado à percepção dos traços suprassegmentais da fala, uma vez que envolve as habilidades em perceber e armazenar os estímulos acústicos não verbais, a percepção do estímulo temporal deficitária pode levar a um baixo desempenho das habilidades de leitura e aprendizagem em geral $^{(2)}$. Tal hipótese foi confirmada neste estudo, no qual observouse associação entre o mau desempenho em ordenação temporal e na $\mathrm{CF}$, habilidade considerada uma das precursoras da leitura.

Uma investigação(14) ${ }^{(14}$ concluiu que a dificuldade envolvendo processamento temporal seria expressa por uma habilidade limitada em identificar e analisar "elementos acústicos curtos" como as consoantes, que são caracterizadas pela rápida transição de formantes, o que levaria a uma dificuldade em associar letras a sons específicos. Pondera-se, assim, que o processamento temporal exerce um papel importante nas habilidades de CF.

A associação encontrada entre a alteração no processamento temporal e baixo desempenho em CF indica a 
necessidade de serem inclusos treinos de processamento temporal no planejamento terapêutico de crianças com alterações de leitura e escrita e na $\mathrm{CF}$, trazendo benefícios no desenvolvimento da $\mathrm{CF}$ e, por sua vez, nas habilidades de leitura e escrita dela dependentes.

O presente estudo traz uma importante contribuição a respeito do papel que as habilidades temporais podem ter no desempenho na $\mathrm{CF}$, o que pode contribuir para novos caminhos no processo terapêutico nas alterações de leitura e escrita.

\section{CONCLUSÃO}

Houve associação entre o desempenho nos testes auditivos temporais e na CF. Os dados encontrados trazem reflexões no sentido de incluir a avaliação dos padrões temporais dentre os procedimentos utilizados na avaliação dos indivíduos com alterações de leitura e escrita.

* MSC e RMMC contribuiu com o desenho do estudo, interpretação de resultados e contribuição na discussão. AJCS e SGGS contribuiu com a coleta de dados, desenho do estudo, interpretação de resultados, contribuição na discussão e redação do artigo. DCA contribuiu com a interpretação de resultados, contribuição na discussão e redação do artigo.

\section{REFERÊNCIAS}

1. Gil D, Almeida CC, Phee AM, Artoni AL, Pellogia CC, Antunes F, et al. Efeito do treinamento auditivo para a percepção musical nos testes de padrão de frequência e duração. Acta WHO. 2000;19(2):64-7.
2. Balen AS, Boeno MRM, Liebel G. A influência do nível socioeconômico na resolução temporal em escolares. Rev Soc Bras Fonoaudiol. 2010;15(1):7-13.

3. Perez AP, Pereira LD. O teste gap in noiseem crianças de 11 e 12 anos. Pro Fono. 2010;22(1):7-12.

4. Germano GD, Pinheiro FH, Capellini SA. Desempenho de escolares com dislexia do desenvolvimento em tarefas fonológicas e silábicas. Rev CEFAC. 2009;11(2):213-20.

5. Soares AJC, Cárnio MS. Consciência fonêmica em escolares antes e após oficinas de linguagem. J Soc Bras Fonoaudiol. 2012;24(1):69-75.

6. Engelmann L, Ferreira MIDC. Avaliação do Processamento auditivo em crianças com dificuldades de aprendizagem. Rev Soc Bras Fonoaudiol. 2009;14(1):69-74.

7. Murphy CFB, Schochat E. Correlações entre leitura, consciência fonológica e processamento temporal auditivo. Pro Fono. 2009;21(1): 13-8.

8. Neves IF, Schochat E. Maturação do processamento auditivo em crianças com e sem dificuldades escolares. Pro Fono. 2005;17(3):311-20.

9. Soares AJC, Sanches SGG, Neves-Lobo IF, Carvallo RMM, Matas CG, Cárnio MS. Potenciais evocados auditivos de longa latência e processamento auditivo central em crianças com alterações de leitura e escrita: dados preliminares. Arq Int Otorrinolaringol. 2011;15(4):486-91.

10. Pinheiro FH, Capellini SA. Treinamento auditivo em escolares com distúrbio de aprendizagem. Pro Fono. 2010;22(1):49-54.

11. Frota S, Pereira LD. Processos temporais em crianças com déficit de consciência fonológica. Rev Iberoam Educac. 2004;33(9):1-9.

12. Moojen S, Lamprecht R, Santos RM, Freitas GM, Brodacz R, Siqueira $\mathrm{M}$, et al. Consciência fonológica: instrumento de avaliação sequencial. São Paulo: Casa do Psicólogo; 2003.

13. Balen AS, Boeno MRM, Liebel G. A influência do nível socioeconômico na resolução temporal em escolares. Rev Soc Bras Fonoaudiol. 2010;15(1):7-13.

14. Habib M. The neurological basis of developmental dyslexia an overview and working hypothesis. Brain. 2000;12(3):2373-99. 